\title{
Interanimal task transfer as a function of dosage of brain and liver RNA injections
}

\author{
G. L. HOLT and B. E. MILLER \\ Eastern Illinois University, Charleston, Illinois 61920
}

\begin{abstract}
The effects of three different dosages of "trained" brain and liver RNA were tested for specific interanimal task transfer. Nine rats were trained to criterion in an operant chamber to approach a food cup when a click was sounded. Thirty-six donor-isolated RNA extractions were taken from the brains and livers of these rats and subsequently divided into three dosages $1.3 \mathrm{mg}$, $.6 \mathrm{mg}$, and $1.2 \mathrm{mg}$ ) within each of four treatment groups: RNase-treated brains and liverscontrol brains (CB) and control livers (CL), and non-RNase-treated brains and livers-trained brains (TB) and trained livers (TL). These 36 trained donor extracts were separately injected intraperitioneally into 36 naive rats. Those rats receiving injections of "trained" RNA from either brain or liver showed a significant $(p<.01)$ tendency to respond more frequently than did RNase-treated controls. The strength of the response transferred was positively related to the quantity of RNA injected, with higher dosages producing better performance.
\end{abstract}

Past experiments have attempted to implicate RNA as having some functional role in the processes of learning and memory. McConnell, Jacobson, and Kimble (1959) showed that when trained planarians were cut in half and allowed 4 weeks for regeneration, there appeared a high retention of the shock avoidance tendency by both the head and tail parts. These results could be viewed as suggesting that memory may not be confined to any one part of the nervous system. McConnell (1982) also showed that planarians that were fed fragments of conditioned worms acquired that conditioned response more rapidly than did controls receiving fragments of naive worms. This was the first suggestion that memory (a learned response) may be enhanced by transferring some unknown substance from one organism to another. However, the transferring agent was not identified.

In further attempts to identify the memory transfer agent, Hýden and Egyházi (1962) demonstrated changes in the molecular structure of nuclear RNA in a trial-anderror experiment involving rats and a tightrope wirewalking task. Later, Hýden and Egyházi (1963) found that the learning process produced changes in glial RNA, showing a higher adenine and lower guanine content and an increase in the amount of RNA per nerve cell in cortical neurons. These changes in structure, content, and amount of RNA modecules of neural cells through learning may suggest that learning and/or memory may be a function of molecular changes in the RNA. However, these changes were not permanent, and much research is needed to relate them properly to memory storage. Concurrent with these studies, Zelman, Kabat, Jacobson, and McConnell (1962) successfully transferred a classical conditioning response by injecting RNA from "trained" planarians ("trained RNA") into naive planarians. This experiment showed that when RNA extracts from the whole "trained" planarian were fed to naive worms, the recipient planarians demonstrated a higher tendency to respond than did naive controls. This study, along with those by Hýden and Egyházi, was among the first to suggest RNA as a transferring agent of memory.

Although these early experiments were inconclusive due to inadequate controls for contaminating effects of protein, DNA, and other amino acids in the RNA extracts, they attracted much attention and speculation as to the potential role of RNA in memory. Cook, Davidson, Davis, Griffin, and Fellows (1963) showed enhancement of learning in rats on a shock avoidance task by intraperitoneal injections of yeast RNA. This shock avoidance seemed to indicate that the intrinsic property of the RNA molecule may increase learning ability. Using more complex learning problems of the Hebb-Williams maze, Ison and Taplin (1966) showed that yeast RNA injections $(100 \mathrm{mg} / \mathrm{kg})$ led to increased performance. Brown (1966) also showed that chronic treatment with yeast RNA produced an increase in response rate during both acquisition and extinction of a leverpressing response on a 15-sec reinforcement schedule. However, yeast RNA is a conglomerate and was suggested to have had contaminants or some RNA by-products that may have been responsible for the effect. One conclusion that may be drawn from the research reviewed above suggests that RNA may play some role in learning and/or memory. The possibility that RNA is only indirectly responsible for creating the effect perhaps by acting as an activating element for some other response center in the brain, would appear to be negated by the research of Babich, Jacobson, Bubash, and Jacobson (1965). They found that magazine training could be transferred in rats by injecting "trained-brain" RNA into naive recipients using a "naive" RNA injection as a control.

There have been negative findings reported as well. 
Luttges, Johnson, Buck, Holland, and McGaugh (1966) failed to obtain a transfer effect when RNA was taken from brains of trained mice using several different tasks, motivation variables, brain preparation techniques, and modes of injection. In addition, when RNA was labeled with P-32, Eist and Seal (1965) found little evidence of P-32-labeled RNA reaching the brain after injection. However, there were few controls in this experiment to eliminate the effects of contaminants in the extracts prior to injections or the potential effects of P-32 on the RNA. Byrne, Samuel, Bennett, Rosenzweig, and Wasserman (1966) also failed to achieve the transfer effect using several tasks such as acquisition of an approach response, learning of a brightness discrimination task, learning a T-maze using food reward, and conditioning of an emotional response using shock avoidance. Further, Gordon, Deanin, Leonhart, and Gwynn (1966), Gurowitz (1968), and Hoffman, Stewart, and Bhagavan (1967) failed to show enhancement in learning by intraperitoneally injecting RNA from magazine-trained rats into naive rats.

Despite these failures to show transfer, there are those who have found interanimal RNA transfer (Essman \& Lehrer, 1967) to be achieved in a learning enhancement experience. Frank, Stein, and Rosen (1970) also found RNA transfer after injecting RNA from shock- or stress-trained donors into naive mice. RNA from both the brain and liver of stress-trained mice were used, with the liver being used to supply high concentrations of nonspecific RNA. Although positive interanimal transfer was achieved, the results were interpreted in terms of stress rather than memory transfer. A "naive" RNA control was the only control used to attempt isolation of the "RNA effect." Using several biochemical and subject control procedures not used by earlier researchers in this area, Miller and Holt (1977) found positive interanimal transfer of a discriminative food magazine approach response using intraperitoneal injections of both liver and brain extracts. Later, Holt and Bentz (Note 1) showed interanimal memory transfer of a discriminated operant using discriminated stimulus cycles of light-and-tone and dark-and-tone sequences as cues for a barpress response. Results showed that both brain and liver RNA recipients showed significant interanimal memory transfer, whereas ribonuclease- (RNase-) treated brain and liver controls showed no transfer effect.

Most of the research reviewed above has been based upon results obtained from RNA extracted from the entire organism or brain homogenates, which may or may not have contained noncontaminated RNA. The majority of research has dealt with learning enhancement rather than specific task transfer. The major difficulty in this area of research has been the deficit of controls for contaminating elements such as protein and DNA, as well as a host of other contaminants whose molecular size is smaller than that of RNA.
The present research was designed to determine whether a specific response could be transferred through injection of "trained" RNA using such control methods as dialysis to remove smaller molecules and RNase to indicate the action of protein or some other contaminant. Whether RNA is the actual storage mechanism or merely an activating element for some other memory storage center in the brain is a question to be answered only through additional research. The following experiment has been designed to show the transfer effects of three different dosages of brain and liver RNA injected intraperitoneally into naive recipients from specific task-trained donors. Thus, relative memory storage potential of the liver as well as the brain and dosage response relationships will be determined.

\section{METHOD}

\section{Subjects}

Forty-five male Sprague-Dawley rats weighing approximately $300 \mathrm{~g}$ each were maintained at $80 \%$ ad-lib weight.

\section{Apparatus}

The apparatus consisted of four basic standard GraysonStadler operant chambers, $27.0 \times 22.5 \times 19.5 \mathrm{~cm}$. The food cup was $5 \mathrm{~cm}^{2}$ and located $.5 \mathrm{~cm}$ above the chamber floor. The boxes had both manual and subject-operated magazines.

\section{Preliminary Training}

Following a 48 -h food-deprivation schedule, nine subjects were trained in the operant chamber to go to the food cup at the click of the food magazine. Preliminary training was terminated when the trained rats approached the food cup and put their nose inside from anywhere within the chamber within $5 \mathrm{sec}$ following the click for at least 3 consecutive days. All other subjects were maintained at $80 \%$ of ad-lib weight throughout the duration of the experiment.

\section{Procedure}

On the last day of preliminary training, the nine trained rats were sacrificed by an intraperitoneal injection of sodium phenobarbitol $(.75 \mathrm{cc})$. They were then decapitated, and the brain and liver were removed. The average weight of liver tissue used was $2.3 \mathrm{~g}$. The entire brain was used from each subject. Each tissue sample was then coded according to its donor. RNA was then extracted from the tissues, using the following procedure.

The tissue was placed in a cold mortar to which was added $10 \mathrm{ml}$ of $90 \%$ phenol and $10 \mathrm{ml}$ of isotonic saline. The tissue was ground for approximately $5 \mathrm{~min}$ and then centrifuged at $16,000 \mathrm{rpm}$ for $30 \mathrm{~min}$. The aqueous phase was carefully drawn off to avoid contamination with phenol in the interphase. The aqueous phase was then brought up to a concentration of $.1-\mathrm{M} \mathrm{MgCl}_{2}$, and two volumes of cold ethanol were added to precipitate the RNA. Precipitation time was approximately $15 \mathrm{~min}$. The solution was centrifuged at $6,000 \mathrm{rpm}$ for $15 \mathrm{~min}$. The supernatant liquid was then poured off and rinsed twice with ethanol. The remaining ethanol was evaporated off, and the RNA was dissolved in $5 \mathrm{ml}$ of isotonic saline. The RNA from each donor was kept separated throughout the experiment according to donor, liver, and brain tissue. After extraction, the RNA was dialyzed against normal saline for $24 \mathrm{~h}$ After dialysis, the amount of RNA was determined by the optical density of $260 \mathrm{MU}(\mathrm{CP}=7,450$ in $.2-\mathrm{M} \mathrm{NaCl})$, yielding an average RNA sample of $4.1 \mathrm{mg} /$ tissue mass. The RNA from each sample was divided into two portions, yielding two samples of brain and two samples of liver RNA from each donor. One 
portion from each donor, for each tissue, was then treated with RNase and incubated for $12 \mathrm{~h}$ at $37^{\circ} \mathrm{C}$. After treatment with RNase, spectrophotometric analysis revealed negligible results.

The procedure yielded 36 extractions, with 4 extractions taken from each of the nine donors. Each donor thus produced one extraction for each of the four groups as follows: one extract of non-RNase-treated brain RNA (TB), one extract of RNase-treated brain RNA (CB), one extract of non-RNasetreated liver RNA (TL), and one extract of RNase-treated liver RNA (CL).

Each dose of RNA was placed in a syringe and coded as to donor, dosage, and treatment of RNA. A double-blind procedure was employed with 36 naive rats receiving an intraperitoneal injection from one of the syringes. Of the 36 rats injected, 3 received injections of $.3 \mathrm{mg}$ of non-RNase-treated brain RNA, 3 received $.6 \mathrm{mg}$ of non-RNase-treated brain RNA, 3 received $1.2 \mathrm{mg}$ of non-RNase-treated brain RNA, 3 received $.3 \mathrm{mg}$ of RNase-treated brain RNA, 3 received $.6 \mathrm{mg}$ of RNase-treated brain RNA, and 3 received $1.2 \mathrm{mg}$ of RNase-treated brain RNA. Three rats received injections of $.3 \mathrm{mg}$ of non-RNase-treated liver RNA, three received injections of $.6 \mathrm{mg}$ of non-RNase-treated liver RNA, and three received injections of $1.2 \mathrm{mg}$ of non-RNasetreated liver RNA, three received injections of $.3 \mathrm{mg}$ of RNasetreated liver RNA, three received injections of $.6 \mathrm{mg}$ of RNasetreated liver RNA, and three received injections of $1.2 \mathrm{mg}$ of RNase-treated liver RNA yielding 12 groups of three subjects in each group.

Prior to injection, the subjects were deprived of food for $28 \mathrm{~h}$. The subjects were placed in operant chambers for $5 \mathrm{~min}$ before a series of clicks produced by the operation of an empty food magazine were delivered. These clicks were spaced no less than 1 min apart. Five test sessions were given at 4, 6, 8, 22, and $24 \mathrm{~h}$ after injection, following the procedure of Babich et al. (1965). Each subject received 25 trials/test session making a total of 125 trials/subject over the five test sessions. Following the third session, all subjects were fed $5 \mathrm{~g}$ of Purina Lab Chow.

A response on a test trial was counted only if the subject placed its nose inside the food cup within 5 sec after the click. Trials were given only when the animal was facing away from the cup by 90 deg or more and located at least a body length away from the cup, when the animal was not already approaching the cup area. During testing, food powder was sprinkled lightly over the floor to avoid responses based on residual odors, and the food cup was cleaned prior to each test session.

\section{RESULTS}

A factorial analysis of variance was performed on the response scores for each subject. Table 1 shows the main effects of dosage level $[F(2,24)=21.75, p<.01]$ and "trained" liver and brain RNA $[F(3,24)=29.02$, $p<.01]$ to be significant at the $p<.01$ level. A high correlation between the number of responses and dosage

Table 1

Analysis of Variance for Transfer Effect as a Function of Dosage and RNA Condition (Conditioned Brain RNA, RNase-Treated Brain RNA, Conditioned Liver RNA, RNase-Treated Liver RNA)

\begin{tabular}{lrrrl}
\hline \multicolumn{1}{c}{ Source } & SS & df & MS & \multicolumn{1}{c}{ F } \\
\hline Dosage & 1283.2 & 2 & 641.6 & $21.75^{*}$ \\
RNA Condition & 2567.9 & 3 & 856.0 & $29.02^{*}$ \\
Dosage by Condition & 2256.3 & 6 & 376.1 & 12.75 \\
Within Cells & 707.6 & 24 & 29.5 & \\
\hline
\end{tabular}

${ }^{*} p<.01$.

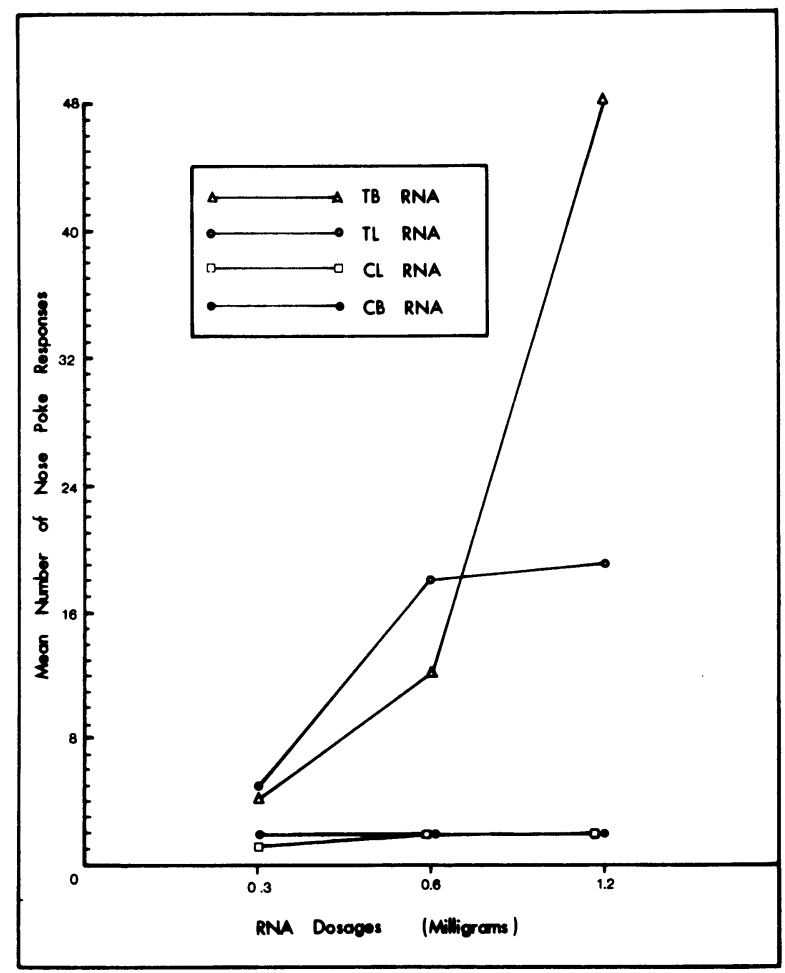

Figure 1. Mean number of nose-poke responses as a function of RNA dosages $(.3, .6$, and $1.2 \mathrm{mg})$ for four recipient groups receiving trained donor brain and liver extract treatments as follows: non-RNase-treated brain RNA (TB), non-RNase-treated liver RNA (TL), RNase-treated liver RNA (CL), and RNasetreated brain RNA (CB).

was noted for both conditioned brain and conditioned liver RNA ( $\mathrm{r}=.9, \mathrm{r}=.8$, respectively).

Figure 1 shows the mean number of nose poke responses as a function of three RNA dosages $(.3, .6$, and $1.2 \mathrm{mg}$ ) and four separate treatment conditions: RNase-treated brains and livers (CB and CL, respectively) and non-RNase-treated brains and livers (TB and TL, respectively). Both brain and liver groups injected with trained RNA but treated with RNase to break up RNA showed no transfer beyond chance levels regardless of dosage. However, both the brain and liver groups not treated with RNase showed significant untrained task transfer, with greater transfer effects resulting from larger RNA dosages (.6 and $1.2 \mathrm{mg}$ ).

The highest dosage $(1.2 \mathrm{mg})$ resulted in over twice the frequency of interanimal task transfer for brain than for liver tissues. This is not true for lower dosages (.3 and $.6 \mathrm{mg}$ ) with which brain tissue produced slightly lower transfer effects.

\section{DISCUSSION}

Much early research has suggested that RNA plays some role in learning and memory storage (Babich et al., 1965; Hýden \& Egyházi, 1962, 1963, 1964; McConnell, 1962; Zelman et al., 1962), whereas others have been unable to show learning 
enhancement and task transfer through RNA injections (Luttges et al., 1966).

There have been few experiments reported that have been designed to test for specific interanimal task transfer from RNA extracts of tissues other than the brain. There has been no prior research reported that determined the strength of the memory transfer as related to dosage of RNA extract.

This experiment has added dosage level as one additional parameter in the study of interanimal specific task transfer while maintaining replicability of the task-transfer phenomenon. Several control methods were employed, such as dialysis to remove molecules smaller than RNA, RNase to reveal any effects of other contaminating agents that might affect the transfer process, and the double-blind procedure.

These results suggest that too small a dosage may not make the transfer effect evident, whereas higher dosages produce strong interanimal transfer effects. The current study suggests that the failure of prior research to produce interanimal memory transfer might be due to inadequate dosage of RNA, whereas research that did show transfer may have used RNA samples of higher dosage. It is also suggested that RNase, DNase, and proteinase controls should be used in future research of this nature to further support the above findings that RNA is at least one viable transfer agent of stored memories.

\section{REFERENCE NOTE}

1. Holt, O. L., \& Bentz, G. Interanimal memory transfer of a discriminated barpress response. Manuscript submitted for publication, 1983.

\section{REFERENCES}

Babich, F. R., Jacobson, A. D., Bubash, S., \& Jacobson, A. Transfer of a response to naive rats by injection of ribonucleic acid extracted from trained rats. Science, 1965, 149, 656-657.

Brown, H. Effect of ribonucleic acid (RNA) on the rate of lever pressing in rats. Psychological Record, 1966, 16, 173-176.

Byrne, W., Samuel, D., Bennett, E., Rosenzweig, M., \& Wasserman, E. Memory transfer. Science, 1966, 153, 658-659.

Coox, L., Davidson, A., Davis, D., Griffin, H., \& Fellows, E. Ribonucleic acid: Effect on conditioned behavior in rats. Science, 1963, 140, 268-269.

Eist, H., \& Seal, U. Permeability of the blood-brain barrier (BBB) and blood-cerebrospinal fluid barrier (BLB) to C14 tagged ribonucleic acid (RNA). American Journal of Psychiatry, 1965, 122, 584-586.

Essman, J. A., \& Lehrer, G. M. Facilitation of maze performances by "RNA extract" from maze-trained mice. Federation Proceedings, 1967, 26, 263.

Frank, B., Stein, P. G., \& Rosen, J. Interanimal "memory" transfer: Results from brain and liver homogenates. Science, $1970,169,399-402$.

Gordon, M., Deanin, G., Leonhart, H., \& Gwynn, R. RNA and memory: A negative experiment. American Journal of Psychiatry, 1966, 10, 1174-1178.

Gurowitz, E. Some effects of brain homogenates on behavior. Psychological Report, 1968, 23, 899-910.

Hoffman, R. F., Stewart, C. W., \& Bhagavan, H. N. Failure to transfer a learned response in rats using a brain extract containing RNA. Psychonomic Science, 1967, 9, 151-152.

HÝden, H., \& EGYHÁzi, E. Nuclear RNA changes of nerve cells during a learning experiment in rats. Proceedings of the National Academy of Science in the United States, 1962, 48, 1366-1373.

HYdEN, H., \& EaYhÁzI, E. Olial RNA changes during a learning experiment with rats. Proceedings of the National Academy of Science in the United States, 1963, 49, 618-624.

HÝden, H., \& EGYHÁz, E. Change in RNA content and base construction in cortical neurons of rats in a learning experiment involving transfer of handedness. Proceedings of the National Academy of Sciences in the United States, 1964, 52, 1030; 1035.

Ison, J. R., \& TAPLIN, P. Reagent grade yeast RNA injections and rat performance on the Hebb-Williams maze. Psychonomic Science, 1966, 6, $495-496$.

Luttges, M., Johnson, T., Buck, C., Holland, J., \& McGaugh, J. An examination of "transfer of learning" by nucleic acid. Science, 1966, 151, 834-837.

McConneld, J. V. Memory transfer through cannibalism in planarians. Neuropsychiatrist, 1962, 3 (Supplement 1), 542-548.

McConnell, J. V., Jacobson, A. L., \& Kimble, D. P. The effects of regeneration upon retention of a conditioned response in planarians. Journal of Comparative and Physiological Psychology, 1959, 52, 1-5.

Mille R, B. E., \& Holt, G. L. Memory transfer in rats by injection of brain and liver RNA. Journal of Biological Psychology, $1977,19,1$.

Zelman, A., Kabat, L., Jacobson, R., \& McConnell, J. V. Transfer of training through injection of "conditioned" RNA into untrained planarians. Worm Runners' Digest, 1962, 5, 14-19. 